\title{
Corilagin sensitizes epithelial ovarian cancer to chemotherapy by inhibiting Snail-glycolysis pathways
}

\author{
LUOQI JIA $^{1 *}$, JIAYI ZHOU ${ }^{1 *}$, HONGBO ZHAO ${ }^{1}$, HONGYAN JIN $^{1}$, MINZHI LV ${ }^{2}$, NAIQING ZHAO ${ }^{2}$, \\ ZHIZHONG ZHENG ${ }^{3}$, YILING LU ${ }^{4}$, YANLIN MING $^{3}$ and YINHUA YU ${ }^{1}$ \\ ${ }^{1}$ Department of Gynecology, Obstetrics and Gynecology Hospital of Fudan University, \\ Shanghai Key Laboratory of Female Reproductive Endocrine-Related Diseases, Shanghai 200090; \\ ${ }^{2}$ Deparment of Biostatistics, School of Public Health, Fudan University, Shanghai 200011; \\ ${ }^{3}$ Key Laboratory of Xiamen City for Plant Introduction and Quarantine and Plant Products, \\ Xiamen Overseas Chinese Subtropical Plant Introduction Garden, Xiamen, Fujian 361002, P.R. China; \\ ${ }^{4}$ Department of Systems Biology, The University of Texas, MD Anderson Cancer Center, Houston, TX 77030, USA
}

Received March 26, 2017; Accepted July 26, 2017

DOI: 10.3892/or.2017.5886

\begin{abstract}
We identified that corilagin is a major component extracted from a well-known hepatoprotective and antiviral medicinal herb, Phyllanthus niruri L with antitumor activity. Our previous study found that corilagin inhibited the growth of ovarian cancer cells via the TGF- $\beta / A K T / E R K$ signaling pathways. Recently, we demonstrated that corilagin enhanced the sensitivity of ovarian cancer cells to chemotherapy. Ovarian cancer cell lines, SKOv3ip, Hey and HO-8910PM-Snail, were treated with different concentrations of corilagin in combination with paclitaxel and carboplatin. Corilagin distinctly enhanced the inhibitory effects of paclitaxel and carboplatin. To understand the mechanisms involved in the chemo-sensitization by corilagin, we performed reverse phase protein array analysis to determine the signaling networks induced by corilagin. We observed that both paclitaxel and carboplatin upregulated the expression levels of several apoptotic and death-related proteins, such as caspase 3, caspase 7 and PDCD4, which were further enhanced when combined with corilagin. Meanwhile, corilagin induced distinct pathways to paclitaxel and carboplatin treatment. We also performed isobaric tags for relative and absolute quantitation proteomics analysis in corilagen-treated
\end{abstract}

Correspondence to: Professor Yinhua Yu, Department of Gynecology, Obstetrics and Gynecology Hospital of Fudan University, 128 Shen Yang Road, Shanghai 200090, P.R. China

E-mail: yin_hu_a@hotmail.com

*Contributed equally

Abbreviations: EMT, epithelial-mesenchymal transition; SRB, sulforhodamine B; RPPA, reverse phase protein array; iTRAQ, isobaric tags for relative and absolute quantitation; ECAR, extracellular acidification rate; OM, oligomycin; 2-DG, 2-deoxyglucose; IC, inhibitory concentration

Key words: corilagin, Snail, glycolysis, ovarian cancer, chemotherapy ovarian cancer cells. This analysis indicated that corilagin is mainly involved in the glycolysis pathway. Seahorse XF96 extracellular acidification rate analysis confirmed that corilagin inhibited glycolysis by downregulation of CD44 and STAT3. In summary, our observations indicate that corilagin sensitized epithelial ovarian cancer cells to paclitaxel and carboplatin treatment by primarily inhibiting Snail-glycolysis pathways. Corilagin is a herbal medicine with low toxic effects to normal cells, particularly hepatoprotective, and may be an ideal complimentary medicine when combined with highly toxic chemotherapeutic agents.

\section{Introduction}

Corilagin $\left(\mathrm{C}_{27} \mathrm{H}_{22} \mathrm{O}_{18}\right)$, a gallotannin found in many plants, is a major active component of many ethnopharmacological plants, such as Phyllanthus niruri L., P. emblica L. and $P$. urinaria L. Corilagin was first isolated in 1951 by Schmidt and Lademann from Divi-divi (Caesalpina coriaria) plants (1). In the past few decades, corilagin has been reported to display several pharmacological activities, including antioxidant (2), hepatoprotective (3), anti-inflammatory (4), neural system protective (5) and cardiovascular protective (6) activities, and has been found to be beneficial in managing type 2 diabetes (7). Recently, its antitumor effects in hepatocellular carcinoma $(8,9)$, ovarian cancer $(10)$ and cholangiocarcinoma (11) have attracted attention.

Since 2005, we screened hundreds of herbs, among which Phyllanthus niruri L. has the highest anticancer potential. The present study further identified that corilagin is a major active component from $P$. niruri L. extracts and has broadspectrum antitumor activity, a better antitumor potential but lower toxicity to normal cells (12). It is effective in retarding the growth of hepatocarcinoma cells by inducing G2/M phase arrest (9); inhibiting ovarian cancer cells via TGF- $\beta / A K T / E R K$ signaling pathways (10); and suppressing cholangiocarcinoma progression through the Notch signaling pathway (11). Recently, we found that corilagin enhances the 
sensitizing effects of chemotherapy drugs in ovarian cancer cells, through Snail epithelial-mesenchymal transition (EMT) and glycolysis pathways.

Therefore, the present study aimed to explore the molecular mechanisms of the sensitizing effects of corilagin in ovarian cancer cells to chemotherapeutic drugs. The present study may provide strong evidence to verify corilagin as a complementary anticancer herbal drug for use in ovarian cancer therapy.

\section{Materials and methods}

Cancer cell lines, $2 D$ and $3 D$ cultures. The human ovarian cancer cell lines SKOv3ip (Skip), OVCAR5, OC316 and Hey were obtained from the MD Anderson Cancer Center (Houston, TX, USA) and were cultured in RPMI-1640 medium supplemented with $10 \%$ fetal bovine serum (FBS). HO8910PM-Snail (HOPM-Snail), a stable Snail-expressing cell line, and its control cell line HO8910PM-vector (HOPM) were cultured in RPMI-1640 medium supplemented with $10 \%$ FBS and $400 \mu \mathrm{g} /$ $\mathrm{ml}$ of G418 as previously described (9). Several ovarian cancer cell lines: Hey, SKOv3ip, HOPM and HOPM-Snail were used for the present study. Hey and SKOv3ip cells were used for cell growth and signaling, HOPM-Snail cells were used for cell growth, signaling and Snail inhibition, and HOPM was used as the parental control. Since Hey and HOPM-Snail cells grow well in Matrigel, these two cell lines were chosen for corilagin inhibition in 3D culture.

3D culture was performed in Matrigel with or without corilagin treatment. Matrigel was used to coat (80-100 $\mu \mathrm{l} /$ well) a pre-cooled 8 -well chamber slide. The chamber slide was placed in a $37^{\circ} \mathrm{C}$ culture incubator for at least $15 \mathrm{~min}$. Cells with a final concentration of $5 \times 10^{4} / \mathrm{ml}$ were put in each well with $4 \%$ Matrigel $(8 / 200 \mu \mathrm{l})$, and maintained in a $37^{\circ} \mathrm{C}$ culture incubator for the indicated time. Corilagin was added after $24 \mathrm{~h}$. Medium and drugs were changed every 3-4 days with 2\% Matrigel. Cells were observed 7-14 days after treatment.

Reagents. Antibodies against pAKT, AKT, pERK, ERK, Snail, STAT3 and CD44 were purchased from Cell Signaling Technology (Danvers, MA, USA), and an anti-GAPDH antibody was purchased from KangChen Biotech, Co., Ltd. (Shanghai, China). Matrigel was purchased from BD Biosciences (San Jose, CA, USA).

Extraction and purification of corilagin. Corilagin was extracted and purified by the Xiamen Overseas Chinese Subtropical Plant Introduction Garden as previously described (12).

Cell proliferation assay. Sulforhodamine B (SRB) was used to detect the effect of drugs on the proliferation of ovarian cancer cell lines, as previously described (10). For each cell line, we tested the different concentrations of each drugs by SRB, and defined the 10, 25 and 50\% inhibitory concentration (IC) for cells and used low (equal to $\mathrm{IC}_{10}$ ), medium (equal to $\mathrm{IC}_{25}$ ) and high (equal to $\mathrm{IC}_{50}$ ) concentrations in all experiments.

Western blot analysis. Cells were seeded into 60-mm plates (1-2x $10^{5} /$ plate) and incubated with corilagin $(20-40 \mu \mathrm{M})$ or dimethyl sulfoxide (DMSO) (as a control) for 24,48 or $72 \mathrm{~h}$.
Cell lysates were harvested with lysis buffer (1\% Triton X-100, $50 \mathrm{mM}$ HEPES, pH 7.4, $150 \mathrm{mM} \mathrm{NaCl}, 1.5 \mathrm{mM} \mathrm{MgCl}_{2}, 1 \mathrm{mM}$ EGTA, $100 \mathrm{mM} \mathrm{NaF}, 10 \mathrm{mM}$ NaPPi and $10 \%$ glycerol, to which $1 \mathrm{mM}$ PMSF, $1 \mathrm{mM} \mathrm{Na} \mathrm{VO}_{4}$, and $1 \mathrm{X}$ protease inhibitor were added before use). For TGF- $\beta$ stimulation assay, HOPMsnail cells were seeded in a $60-\mathrm{mm}$ plate, starved overnight and treated with TGF- $\beta 1$ alone or in combination with corilagin; DMSO was used as the control. Proteins from total cell lysates were resolved using a 10-15\% SDS-PAGE gel and transferred to polyvinylidene difluoride (PVDF) membranes (Millipore, Billerica, MA, USA). The membranes were blocked, washed and incubated with specific primary antibodies, followed by incubation with HRP-conjugated secondary antibodies. The bands were detected with an enhanced chemiluminescence assay (PerkinElmer, Waltham, MA, USA).

Reverse phase protein array (RPPA) analysis. Untreated and corilagin-treated HOPM-Snail cells were used for RPPA analysis at The University of Texas, MD Anderson Cancer Center RPPA Core Facility. We followed the methods described at the following web address: http://www.mdanderson.org/educationand-research/resources-for-professionals/scientific-resources/ core-facilities-and-services/functional-proteomics-rppa-core/ index.html.

Proteomics analysis. Isobaric tags for relative and absolute quantitation (iTRAQ) proteomics analysis (OmicsBean, Shanghai, China) were performed in corilagen-treated ovarian cancer Skip and Hey cells. Untreated cells were used as the control.

Glycolysis analysis. Glycolysis was analyzed using a Seahorse XF96 extracellular flux analyzer (Seahorse Bioscience, Shanghai, China) by real-time measurements of the extracellular acidification rate (ECAR) which is indicative of glycolysis. Cells (Skip and Hey, 5,000 cells/well; HOPM and HOPM-Snail, 8,000 cells/well) were seeded in complete growth medium in the wells of 96-well plates designed for the XF96 with quadruple repeat. Different concentrations of corilagin were added on the following day for $24 \mathrm{~h}$. Cells were incubated in basal glucose-free medium before incubation in a $\mathrm{CO}_{2}$-free incubator after treatment with PEG-GO. The default standard glycolysis stress-test program was selected. Measurements were conducted using final concentrations of $10 \mathrm{mM}$ glucose, $1 \mathrm{mM}$ oligomycin (OM) or $100 \mathrm{mM}$ 2-deoxyglucose (2-DG)

Statistical analysis. Triplicates or duplicates were performed in each experiment. All data were subjected to statistical analysis and were reported as the mean \pm standard deviation. The criterion for statistical significance was taken as $\mathrm{P}<0.05$ using a two-tailed t-test and the count data were tested using Chi-square criterion comparing the frequency of parameters. Analyses were performed using SPSS 15.0 software (SPSS, Inc., Chicago, IL, USA).

\section{Results}

Corilagin inhibits $3 D$ cell culture. Since the advent of routine eukaryotic cell culture $>40$ years ago, the most 

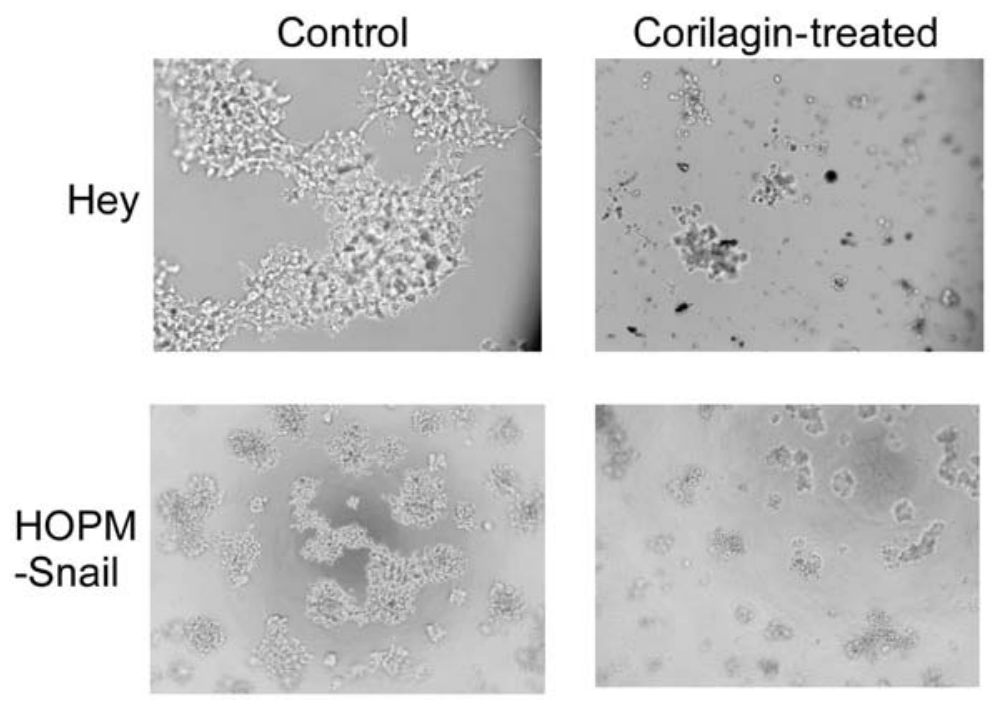

Figure 1. Corilagin inhibits 3D culture of ovarian cancer cells.

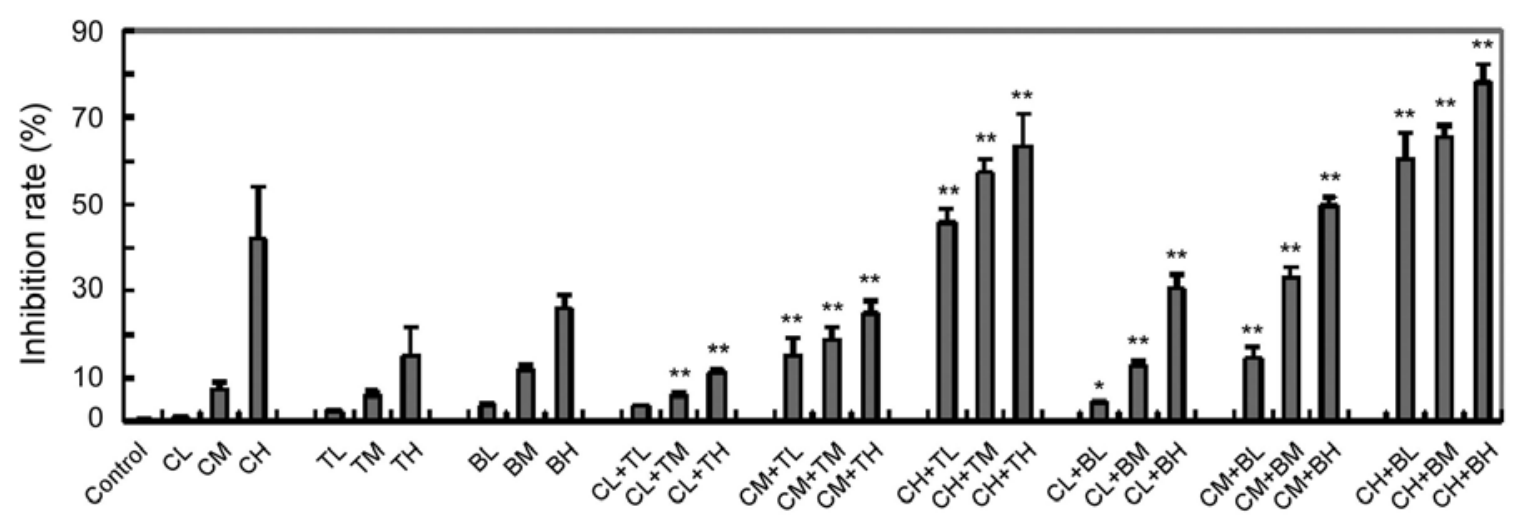

Inhibition rate of corilagin combined with paclitaxel

\begin{tabular}{|l|r|r|r|r|}
\hline & 0 & TL & \multicolumn{1}{l|}{ TM } & \multicolumn{1}{|c|}{} \\
\hline 0 & 0.0010 & 0.0245 & 0.0588 & 0.1538 \\
\hline $\mathrm{CL}$ & 0.0089 & 0.0359 & 0.0602 & 0.1126 \\
\hline $\mathrm{CM}$ & 0.0722 & 0.1506 & 0.1861 & 0.2496 \\
\hline $\mathrm{CH}$ & 0.4198 & 0.4562 & 0.5737 & 0.6365 \\
\hline
\end{tabular}

Inhibition rate of corilagin combined with carboplatin

\begin{tabular}{|l|r|r|r|r|}
\hline & 0 & \multicolumn{1}{|l|}{ BL } & \multicolumn{1}{l|}{ BM } & \multicolumn{1}{l|}{ BH } \\
\hline 0 & 0.0010 & 0.0356 & 0.1173 & 0.2597 \\
\hline $\mathrm{CL}$ & 0.0089 & 0.0428 & 0.1266 & 0.3056 \\
\hline $\mathrm{CM}$ & 0.0722 & 0.1467 & 0.3298 & 0.4974 \\
\hline $\mathrm{CH}$ & 0.4198 & 0.6061 & 0.6556 & 0.7797 \\
\hline
\end{tabular}

Figure 2. Corilagin enhances the inhibitory effects of paclitaxel and carboplatin in ovarian cancer cells. Upper panel: Skip cells were simultaneously treated with low, medium and high concentrations of corilagin (CL, CM, CH); paclitaxel (TL, TM, TH); carboplatin (BL, BM, BH); and their combinations. SRB was used to test cell proliferation; ${ }^{*} \mathrm{p}<0.05,{ }^{* *} \mathrm{p}<0.01$. Lower panel: Inhibition rate of corilagin combined with paclitaxel and corilagin combined with carboplatin.

common substrates for supporting cell growth have been made from polystyrene or glass and have taken the form of a flat two-dimensional surface. As we described previously, in this $2 \mathrm{D}$ culture system, corilagin demonstrated significant inhibition of ovarian cancer cell growth, but had much lower cytotoxicity in normal ovarian surface epithelium cells (10). To overcome some disadvantages of the 2D system, a number of three-dimensional methods have been developed. Matrigel (BD Biosciences) provides a biologically active basement membrane model for in vitro invasion assay and drug toxicity studies (13). We investigated the effects of corilagin in a 3D system with BD Matrigel. As shown in Fig. 1, both Hey and HOPM-Snail cells formed extensive growth colonies and connected bridges in the Matrigel. Corilagin treatment hampered the colony formation in both cell lines, suggesting that corilagin not only inhibited ovarian cancer cell growth, but also affected cell invasion.

Corilagin enhances the inhibitory effects of chemotherapy drugs in ovarian cancer cells. Different concentrations of corilagin were used in combination assays with paclitaxel and carboplatin in ovarian cancer cell lines SKip, Hey and HOPMSnail. Corilagin distinctly increased the inhibitory effects of paclitaxel and carboplatin (Fig. 2). Statistical analysis confirmed that corilagin had an additive effect when combined with chemotherapeutic drugs. There were significant changes between low, medium and high concentrations of corilagin (CL, CM, CH), paclitaxel (TL, TM, TH) or carboplatin (BL, BM, BH). In contrast to treatment with each single drug only, no difference was noted in CL $+\mathrm{TL}$, while significant 


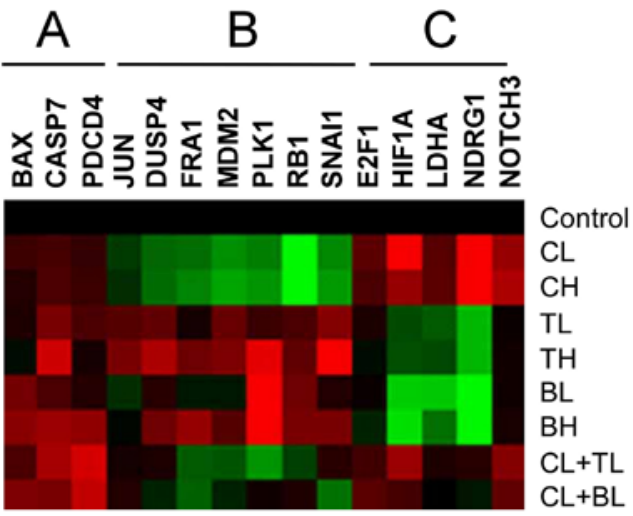

Figure 3. RPPA analysis. HOPM-Snail cells treated with low, high doses of corilagin $(\mathrm{CL}, \mathrm{CH})$; paclitaxel $(\mathrm{TL}, \mathrm{TH})$; carboplatin $(\mathrm{BL}, \mathrm{BH})$ or their combinations. The figure presents a small portion of the results. Red color indicates enhanced expression, green color indicates reduced expression. Untreated cells were used as the control. A, Corilagin enhances apoptotic effects of drugs; B, Corilagin inhibits these proteins, but drugs do not; $\mathrm{C}$, Corilagin increases these proteins, but drugs do not.

differences were noted in $\mathrm{CL}+\mathrm{BL}(\mathrm{p}<0.05)$ and in the other combinations $(\mathrm{p}<0.01)$.

Corilagin acts not only on apoptotic pathways, but also via its distinct pathways. To understand the sensitization mechanisms of corilagin, we performed RPPA analysis to determine corilagin-induced signaling networks, using HOPM-Snail cells, treated with paclitaxel and carboplatin in the presence or absence of corilagin. As presented in Fig. 3, both paclitaxel and carboplatin treatment upregulated the levels of several apoptotic and death proteins, caspase 3, caspase 7, and PDCD4, while combined with corilagin, these apoptotic effects were further enhanced. Meanwhile, corilagin demonstrated distinct pathways to paclitaxel and carboplatin. Corilagin inhibited the expression of Snail, PLK1 and RB1, and enhanced the expression of HIF1A and NDRG1, which were opposite to paclitaxel or carboplatin treatment. All these changes suggest that corilagin acts not only on apoptotic pathways, but also via its distinct pathways.

RPPA results were confirmed by western blot analyses. We found that corilagin treatment downregulated Snail expression in three ovarian cancer cell lines (OVCAR5, OC316 and HOPMSnail) (Fig. 4A-C), particularly in the Snail-overexpressing cell line HOPM-Snail. Corilagin blocked TGF $\beta$-enhanced Snail in HOPM-Snail cells (Fig. 4C). Corilagin decreased the expression of Snail and phospho-ERK, which was not observed following paclitaxel and carboplatin treatments (Fig. 4D). All these results suggest that corilagin is not only an apoptosis inducer, similar to chemotherapeutic drugs, but also has its distinct pathways, such as inhibition of Snail, which has been recognized as an EMT inducer.

Corilagin inhibits the glycolysis pathways via Snail. Our previous study demonstrated that Snail is significantly overexpressed in metastatic lesions, and Snail is a key inducer of EMT in ovarian cancer (14). To understand the effects of corilagin in Snail-EMT pathways, we performed iTRAQ proteomics analysis in corilagen-treated ovarian cancer cells. In total, with FDR $<0.01$ and P-value $<0.05$ cut-off, we identified 108 proteins that were differentially expressed and mainly downregulated ( 28 upregulated, 80 downregulated) by corilagin treatment. The targeted proteins were found to be cytoskeletal proteins and related enzymes (lipase, ATPase and kinase), mainly involved in the glycolysis pathway. Enrichment results of both biological processes and KEGG pathways revealed that several pathways such as glycolysis pathway, focal adhesion, the HIF-1 signaling pathway and proteoglycans

A

B

C HOPM-Snail
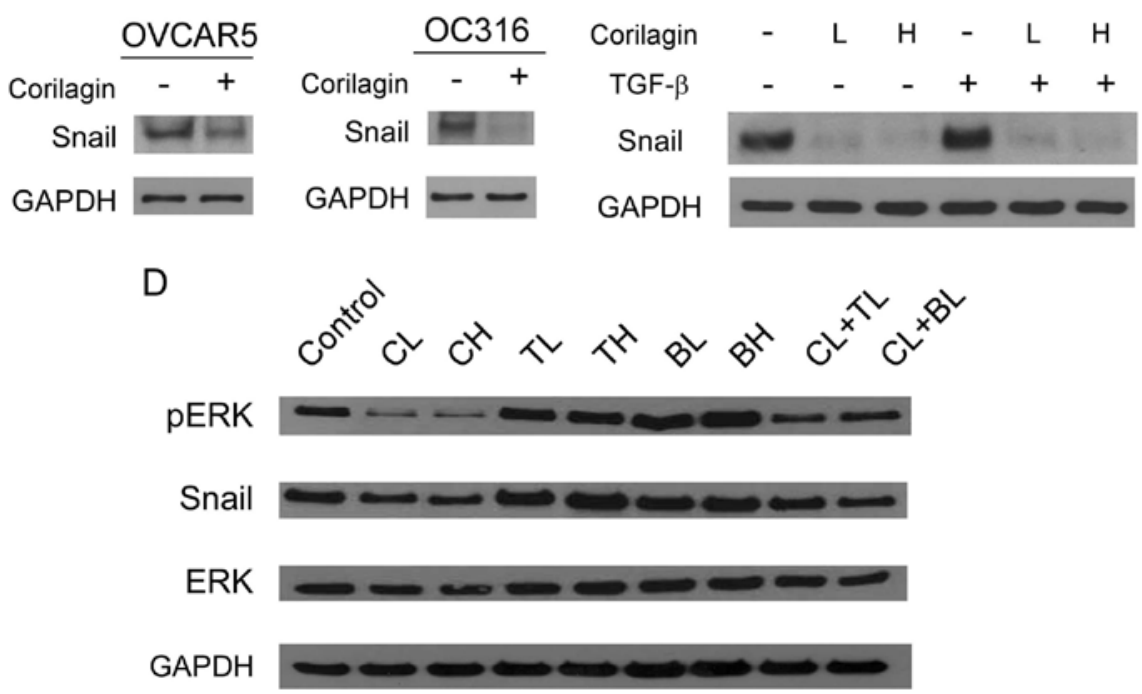

Figure 4. Corilagin inhibits Snail expression in ovarian cancer cells: (A) OVCAR5, (B) OC316, (C) HOPM-Snail. Corilagin concentration in A and B is high, in $\mathrm{C}$ is low and high ( $\mathrm{L}, \mathrm{H})$. (C) HOPM-Snail cells were treated with TGF- $\beta$ for $6 \mathrm{~h}$ after starvation for $24 \mathrm{~h}$. Snail expression was detected by western blotting. GAPDH was used as the control. (D) HOPM-Snail cells were treated by a low and high concentration of corilagin (CL, CH); paclitaxel (TL, TH); carboplatin (BL, BH); and their combinations in a low concentration; untreated cells were used as the control. Phosphorylated ERK (pERK) and Snail were detected by western blotting, and total ERK and GAPDH were also detected as loading controls. 

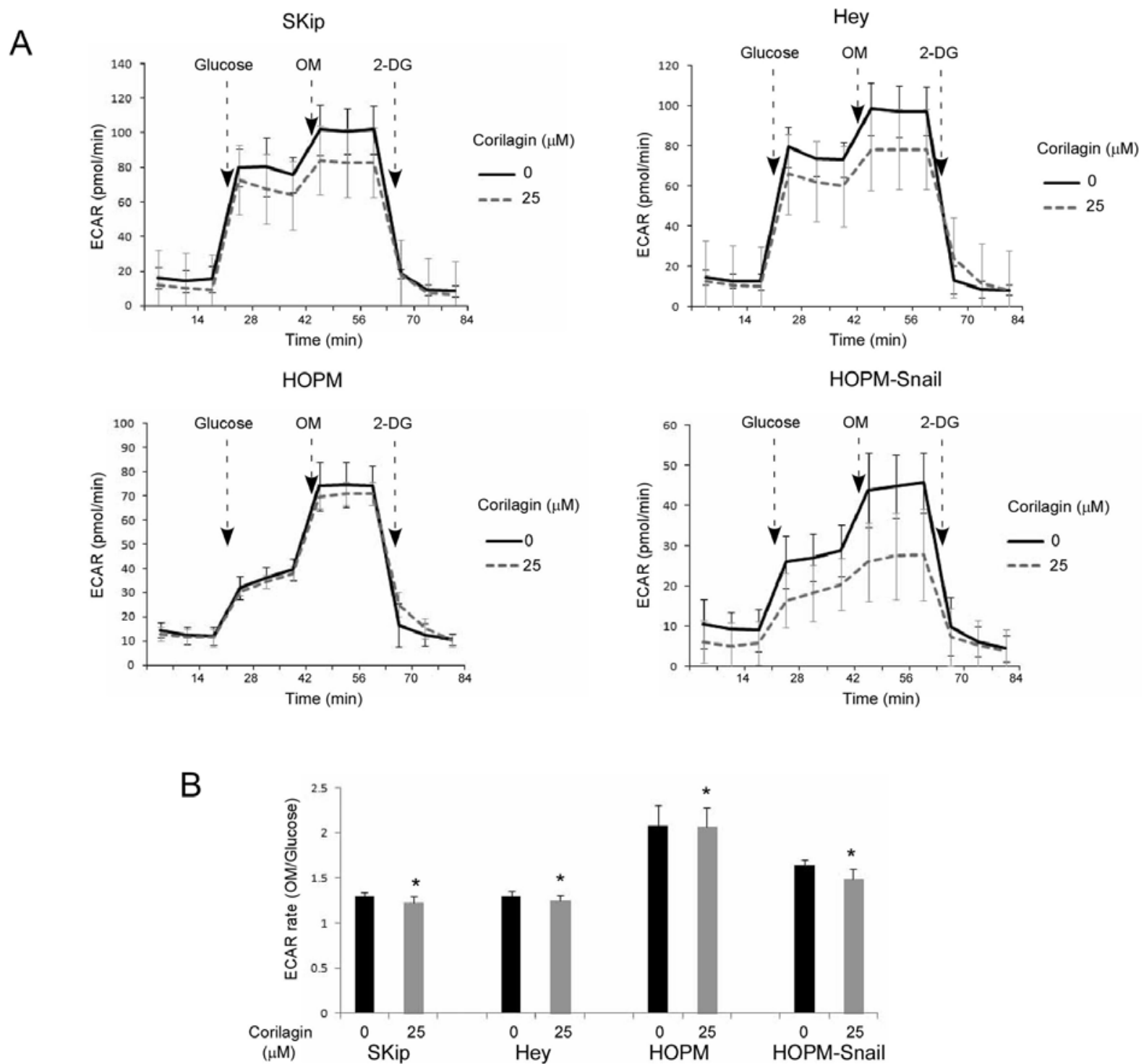

Figure 5. Analysis of glycolysis. Four ovarian cancer cell lines were used for Seahorse XF96 ECAR analysis. Details are described in Materials and methods. (A) ECAR curve. Glycolysis was indicated as the ECAR value expressed as means \pm SEM ( $n=3$ or 4). Dotted arrows indicate the injection of glucose, OM and 2-DG. (B) ECAR rate is presented as the average value of OM treatment divided by the average value of glucose treatment; " $p<0.05$, compared with corilagin untreated cells.

were inhibited. Moreover, corilagen may block glycolysis by inhibiting several key proteins, including ENO1, LDHA, GPI and PGK1; further downregulating CD44, cortactin, STAT3 and filamin.

To the best of our knowledge, increased aerobic glycolysis in cancer cells, termed the Warburg effect, represents a potential targeting strategy for cancer treatment (15). Snail and other transcription factors, such as STAT3 and CD44, are known to regulate glycolysis (16-23), and then further affect tumor growth. To validate the iTRAQ proteomics results, we investigated whether corilagin affects glycolysis. The extracellular acidification rate (ECAR), which indicates the rate of glycolysis (basal glycolysis, maximal glycolytic capacity and glycolytic reserve) (24), was determined using a Seahorse XF96 extracellular flux analyzer (Fig. 5A). The results showed that four ovarian cancer cell lines (Skip, Hey, HOPM and HOPM-Snail) treated with corilagin had a lower ECAR curve when compared with the untreated cells. Compared with

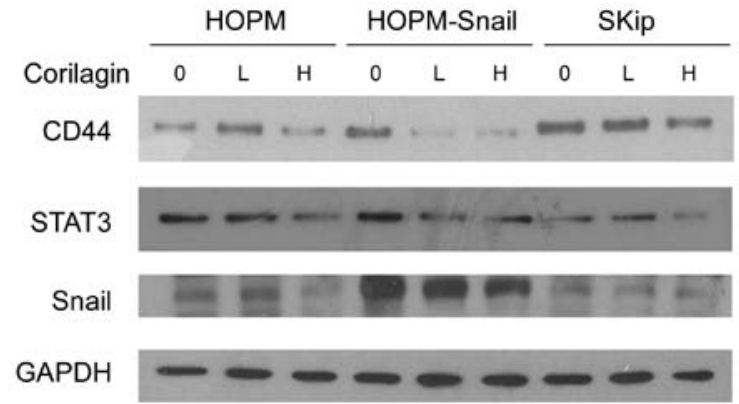

Figure 6. Corilagin inhibits CD44 and STAT3 expression. Three ovarian cancer cell lines (Skip, HOPM and HOPM-Snail) were treated with corilagin, and used for western blot analysis. GAPDH was used as a loading control. L or $\mathrm{H}$, low or high doses of corilagin.

the HOPM parental cells, HOPM-Snail cells showed much higher glycolysis inhibition, suggesting that corilagin inhibits 
glycolysis via Snail. When the ECAR rate was presented as the average value of OM injected divided by the average value of glucose injected, all four corilagin treated cells had a lower rate compared with the untreated cells $(\mathrm{p}<0.05)$ (Fig. 5B).

Western blot analysis also indicated that corilagin did inhibit the expression of CD44 and STAT3 (Fig. 6), both of which have been demonstrated to be crucial for glycolysis regulation (18-23). Corilagin showed strong inhibition of Snail in HOPM-Snail cells, compared with HOPM cells.

\section{Discussion}

Conventional chemotherapeutic agents, such as alkylating agents and Taxol, in ovarian cancer, shrink tumor size but with high toxicity. In addition, repeated treatment with these agents leads tumors to acquire resistance to the chemotherapies. Therefore, it is necessary to discover compounds with growth inhibitory and apoptosis induction properties in cancer cells but causing less or no toxicity in normal cells. Ideally, such compounds could target multiple major cellular signaling pathways in cancer cells. Recent research has focused on herbal medicines, among which, corilagin is a novel anticancer agent. This drug was confirmed to inhibit cancer growth involving multiple pathways (8-11). Corilagin was reported to have the following characteristics: non-toxicity to normal tissues, a wide spectrum of efficacy against multiple cancers, and ability for oral consumption. Importantly, our experiments displayed that corilagin enhanced the effects of paclitaxel and carboplatin in all ovarian cancer cells, being an ideal complementary medicine in cancer therapy. Corilagin presented different mechanisms in ovarian cancer when compared with those of current chemotherapy. In our previous study, we showed that corilagin inhibited TGF- $\beta$ secretion into the culture supernatant of ovarian cancer cell lines. In contrast, a reduction in TGF- $\beta$ secretion was not observed in cancer cells treated with the cytotoxic drug paclitaxel, suggesting that corilagin specifically targets TGF- $\beta$ secretion (10). In the present study, we found that corilagin acts not only on apoptotic pathways, but also via its distinct pathways, such as inhibition of Snail and phospho-ERK (Fig. 4). Conventional drugs, such as paclitaxel and carboplatin, inhibited the phosphorylation of AKT, but did not inhibit the phosphorylation of ERK, and also did not inhibit Snail. These studies may explain why corilagin displays additive effects with chemotherapeutic drugs during treatment.

Recently, numerous studies have focused on cancer drug resistance, and the role of EMT in chemoresistance has emerged. EMT is a highly conserved process in which polarized, immobile epithelial cells lose tight junctions, associated adherence and become migratory mesenchymal cells. Several transcription factors, including the Snail/Slug family, Twist, $\delta E F 1 / Z E B 1$, SIP1/ZEB2 and E12/E47 respond to microenvironmental stimuli and function as molecular switches for the EMT program (25). Among these factors, Snail is the most important one. Our previous study confirmed that Snail is critical for tumor growth and metastasis of ovarian carcinoma (14). Snail causes a metabolic reprogramming, bestows tumor cells with cancer stem cell-like traits and additionally, promotes drug resistance, tumor recurrence and metastasis (25). Kurrey et al found that Snail and Slug are critical for a cancer cell to acquire stem cell-like characteristics toward resisting radiotherapy- or chemotherapy-mediated cellular stress (26). In the present study, we demonstrated that corilagin enhanced chemosensitivity by inhibition of Snail, and also by inhibition of CD44 and Stat3, factors that may relate to cancer stem cell development. This herbal medicine may provide a new strategy to overcome chemoresistance.

Glycolysis is the major source of energy in cancer cells. Warburg et al showed in the 1920s that under aerobic conditions, tumor tissues metabolize $\sim 10$-fold more glucose to lactate in a given time than normal tissues, a phenomenon known as the Warburg effect. However, this increase in aerobic glycolysis in cancer cells is often erroneously thought to occur instead of mitochondrial respiration and has been misinterpreted as evidence for damage to respiration instead of damage to the regulation of glycolysis. In fact, many cancers exhibit the Warburg effect while retaining mitochondrial respiration (15). To the best of our knowledge, TGF $\beta 1$-induced EMT is accompanied by coordinately reduced enzyme expression required to convert glucose into fatty acids, and concomitant enhanced respiration (16). Snail, a transcription factor mediating TGF $\beta 1$-induced EMT, suppresses lipogenesis and favors energy production through carbohydrate-responsive-element-binding protein (ChREBP, a master lipogenic regulator), and fatty acid synthase (FASN) (16). In the present study, corilagin treatment inhibited the ECAR curve, which indicates the rate of glycolysis, in ovarian cancer cells. Compared with HOPM parental cells, HOPM-Snail cells showed much higher glycolysis inhibition when Snail was clearly downregulated by corilagin (Fig. 6), suggesting that the inhibition of glycolysis by corilagin could be mainly through Snail-TGF $\beta$ inhibition.

Proteomics analysis revealed that corilagen may block glycolysis by inhibiting several other key proteins except Snail, such as CD44, cortactin, STAT3 and filamin. We confirmed that corilagin inhibited CD44 and STAT3 in all ovarian cancer cells (Fig. 6). STAT3 acts as a master regulator of cell metabolism, inducing aerobic glycolysis and downregulating mitochondrial activity (20). CD44, a cell surface marker for cancer stem cells, interacts with pyruvate kinase M2 (PKM2) and thereby enhances the glycolytic phenotype of cancer cells. Ablation of CD44 induces glycolysis-to-oxidative phosphorylation transition (21). Inhibition of CD44 also sensitized prostate cancer cells to carboplatin (22). Reduction of STAT3 and CD44 expression has a significant impact on the study of corilagin mechanisms. STAT3 and CD44 may regulate glycolysis by different ways. Inhibition of glycolysis by corilagin could also occur through STAT3 and CD44 pathways, which is a new area to explore.

\section{Acknowledgements}

The present study was supported by funds from the National Natural Science Foundation of China (grant no. 81274149 to Y.M.). The present study was also supported by grants from the Natural Science Foundation of Fujian Province (grant no. 2010D012 to Y.M.), the Xiamen Municipal Science and Technology Innovation Fund Project (grant no. $3502 Z 20101016$ to Y.M.), and the Shanghai Pujiang Program (grant no. 15PJ1400900 to H.Z.). 


\section{References}

1. Schmidt OT and Lademann R: Corilagin, ein weiterer kristallisierter Gerbstoff aus Dividivi. X. Mitteilung über natürliche Gerbstoffe. Justus Liebigs Ann Chem 571: 232-237, 1951 (In German)

2. Wu N, Zu Y, Fu Y, Kong Y, Zhao J, Li X, Li J, Wink M and Efferth T: Antioxidant activities and xanthine oxidase inhibitory effects of extracts and main polyphenolic compounds obtained from Geranium sibiricum L. J Agric Food Chem 58: 4737-4743, 2010.

3. Kinoshita S, Inoue Y, Nakama S, Ichiba T and Aniya Y: Antioxidant and hepatoprotective actions of medicinal herb, Terminalia catappa L. from Okinawa Island and its tannin corilagin. Phytomedicine 14: 755-762, 2007.

4. Zhao L, Zhang SL, Tao JY, Pang R, Jin F, Guo YJ, Dong JH, Ye P, Zhao HY and Zheng GH: Preliminary exploration on anti-inflammatory mechanism of corilagin (beta-1-O-galloyl3,6-(R)-hexahydroxydiphenoyl-d-glucose) in vitro. Int Immunopharmacol 8: 1059-1064, 2008.

5. Dong XR, Luo M, Fan L, Zhang T, Liu L, Dong JH and Wu G: corilagin inhibits the double strand break-triggered NF-kappaB pathway in irradiated microglial cells. Int J Mol Med 25: 531-536, 2010.

6. Duan W, Yu Y and Zhang L: Antiatherogenic effects of Phyllanthus Emblica associated with corilagin and its analogue. Yakugaku Zasshi 125: 587-591, 2005.

7. Gao H, Huang YN, Xu PY and Kawabata J: Inhibitory effect on $\alpha$-glucosidase by the fruits of Terminalia chebula Retz. Food Chem 105: 628-634, 2007.

8. Hau DK, Zhu GY, Leung AK, Wong RS, Cheng GY, Lai PB, Tong SW, Lau FY, Chan KW, Wong WY, et al: In vivo antitumour activity of corilagin on Hep3B hepatocellular carcinoma. Phytomedicine 18: 11-15, 2010.

9. Ming Y, Zheng Z, Chen L, Zheng G, Liu S, Yu Y and Tong Q: Corilagin inhibits hepatocellular carcinoma cell proliferation by inducing G2/M phase arrest. Cell Biol Int 37: 1046-1054, 2013.

10. Jia L, Jin H, Zhou J, Chen L, Lu Y, Ming Y and Yu Y: A potential anti-tumor herbal medicine, corilagin, inhibits ovarian cancer cell growth through blocking the TGF- $\beta$ signaling pathways. BMC Complement Altern Med 13: 33, 2013.

11. Gu Y, Xiao L, Ming Y, Zheng Z and Li W: Corilagin suppresses cholangiocarcinoma progression through Notch signaling pathway in vitro and in vivo. Int J Oncol 48: 1868-1876, 2016.

12. Zheng ZZ, Chen LH, Liu SS, Deng Y, Zheng GH, Gu Y and Ming YL: Bioguided fraction and isolation of the antitumor components from Phyllanthus niruri L. Biomed Res Int 2016 9729275, 2016

13. Lee GY, Kenny PA, Lee EH and Bissell MJ: Three-dimensional culture models of normal and malignant breast epithelial cells. Nat Methods 4: 359-365, 2007.
14. Jin H, Yu Y, Zhang T, Zhou X, Zhou J, Jia L, Wu Y, Zhou BP and Feng Y: Snail is critical for tumor growth and metastasis of ovarian carcinoma. Int J Cancer 126: 2102-2111, 2010.

15. Koppenol WH, Bounds PL and Dang CV: Otto Warburg's contributions to current concepts of cancer metabolism. Nat Rev Cancer 11: 325-337, 2011.

16. Jiang L, Xiao L, Sugiura H, Huang X, Ali A, Kuro-o M, Deberardinis RJ and Boothman DA: Metabolic reprogramming during TGF $\beta 1$-induced epithelial-to-mesenchymal transition. Oncogene 34: 3908-3916, 2015.

17. Dong C, Yuan T, Wu Y, Wang Y, Fan TW, Miriyala S, Lin Y, Yao J, Shi J, Kang T, et al: Loss of FBP1 by Snail-mediated repression provides metabolic advantages in basal-like breast cancer. Cancer Cell 23: 316-331, 2013.

18. Poli V and Camporeale A: STAT3-mediated metabolic reprograming in cellular transformation and implications for drug resistance. Front Oncol 5: 121, 2015.

19. Li J, Liu T, Zhao L, Chen W, Hou H, Ye Z and Li X: Ginsenoside 20(S)-Rg3 inhibits the Warburg effect through STAT3 pathways in ovarian cancer cells. Int J Oncol 46: 775-781, 2015.

20. Demaria M, Giorgi C, Lebiedzinska M, Esposito G, D'Angeli L, Bartoli A, Gough DJ, Turkson J, Levy DE, Watson CJ, et al: A STAT3-mediated metabolic switch is involved in tumour transformation and STAT3 addiction. Aging 2: 823-842, 2010.

21. Nam K, Oh S and Shin I: Ablation of CD44 induces glycolysisto-oxidative phosphorylation transition via modulation of the c-Src-Akt-LKB1-AMPK $\alpha$ pathway. Biochem J 473: 3013-3030, 2016.

22. Li W, Cohen A, Sun Y, Squires J, Braas D, Graeber TG, Du L, $\mathrm{Li} \mathrm{G}, \mathrm{Li} \mathrm{Z}, \mathrm{Xu}$ X, et al: The role of CD44 in glucose metabolism in prostatic small cell neuroendocrine carcinoma. Mol Cancer Res 14: 344-353, 2016.

23. Tamada M, Nagano O, Tateyama S, Ohmura M, Yae T, Ishimoto T, Sugihara E, Onishi N, Yamamoto T, Yanagawa H, et al: Modulation of glucose metabolism by CD44 contributes to antioxidant status and drug resistance in cancer cells. Cancer Res 72: 1438-1448, 2012.

24. Zhou T, Zhang B, Wei P, Du Y, Zhou H, Yu M, Yan L, Zhang W, Nie G, Chen C, et al: Energy metabolism analysis reveals the mechanism of inhibition of breast cancer cell metastasis by PEG-modified graphene oxide nanosheets. Biomaterials 35: 9833-9843, 2014.

25. Wang Y, Shi J, Chai K, Ying X and Zhou BP: The role of Snail in EMT and tumorigenesis. Curr Cancer Drug Targets 13: 963-972, 2013.

26. Kurrey NK, Jalgaonkar SP, Joglekar AV, Ghanate AD, Chaskar PD, Doiphode RY and Bapat SA: Snail and slug mediate radioresistance and chemoresistance by antagonizing p53-mediated apoptosis and acquiring a stem-like phenotype in ovarian cancer cells. Stem Cells 27: 2059-2068, 2009. 